\title{
Espaçamento, densidade e uniformidade de semeadura na produtividade e características agronômicas da soja ${ }^{(1)}$
}

\author{
Maria Cristina Cavalheiro Tourino(2), Pedro Milanez de Rezende ${ }^{(3)}$ e Nilson Salvador ${ }^{(2)}$
}

\begin{abstract}
Resumo - O objetivo do presente estudo foi avaliar o efeito do espaçamento entre linhas $(45$ e $60 \mathrm{~cm}$ ),

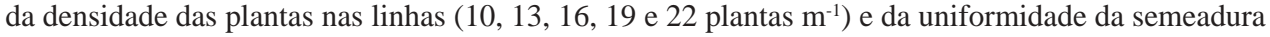
(espaçamentos aceitáveis $25 \%, 35 \%, 60 \%$ e 100\%), sobre a produtividade da soja. O experimento foi conduzido nos anos 1997/1998 e 1998/1999, com a cultivar CAC-1. O delineamento experimental utilizado foi o de blocos ao acaso, com três repetições, com os tratamentos em esquema de parcelas subsubdivididas. Houve aumento na produtividade da soja no espaçamento de $45 \mathrm{~cm}$ com a redução da densidade para 10 plantas $\mathrm{m}^{-1}$, e com a uniformidade de espaçamento entre plantas dentro das linhas acima de $60 \%$. A altura das plantas e o grau de acamamento foram reduzidos significativamente; porém, a porcentagem de sobrevivência das plantas aumentou à medida que ocorreu redução da densidade populacional das plantas. Também foi detectado que o aumento da uniformidade de espaçamento entre as plantas dentro das linhas reduziu-lhes o acamamento.
\end{abstract}

Termos para indexação: Glycine max, acamamento, grãos, rendimento.

\section{Row spacing, plant density and intrarow plant spacing uniformity effect on soybean yield and agronomic characteristics}

\begin{abstract}
The objective of the present study was to evaluate the row spacing (45 and $60 \mathrm{~cm}$ ), plant populational density $\left(10,13,16,19\right.$ and 22 plants $\left.\mathrm{m}^{-1}\right)$ and intrarow plant spacing uniformity (acceptable spacing $25 \%, 35 \%, 60 \%$ and $100 \%$ ) effects on soybean yield. The experiment was carried out in the years 1997/1998 and 1998/1999, using the CAC-1 cultivar. The experimental design was a splitsplit plot, with three replications. There was increase in the soybean yield in the $45 \mathrm{~cm}$-row spacing when plant density was reduced to 10 plants $\mathrm{m}^{-1}$, and with uniformity above $60 \%$. The plants height and lodging were significantly reduced; however, survival was increased as reduction of the density happened. It was also detected that the increase of the spacing uniformity reduced their lodging.
\end{abstract}

Index terms: Glycine max, lodging, grain, yields.

\section{Introdução}

O Brasil é o segundo maior produtor mundial de soja, pois alcança $20,1 \%$ de toda a produção dessa leguminosa no mundo, o que equivale a 31,4 milhões de toneladas (Embrapa, 2000). As áreas de cerrado, por suas condições topográficas e seu clima favoráveis, vêm ocupando posição de destaque no cenário

\footnotetext{
(1) Aceito para publicação em 13 de novembro de 2001.

(2) Universidade Federal de Lavras (Ufla), Dep. de Engenharia, Caixa Postal 37, CEP 37200-000 Lavras, MG. E-mail: mcctouri@ufla.br,salvador@ufla.br

(3) Ufla, Dep. de Agricultura. Bolsista do CNPq. E-mail pmrezende@ufla.br
}

nacional. $\mathrm{O}$ excelente desempenho da cultura nessa região (Mato Grosso do Sul) deve-se à adoção, pelos produtores, de novas tecnologias, principalmente das relacionadas com o fator cultivar resistente a doenças e com maior potencial produtivo (Embrapa, 2000).

A maior expressão do potencial produtivo das cultivares, entretanto, depende das condições do meio onde as plantas irão desenvolver-se. Assim, alterações relacionadas com a população de plantas podem reduzir ou aumentar os ganhos em produtividade, pois essa característica é conseqüência da densidade das plantas nas linhas e do seu espaçamento entre as linhas. A população de plantas é o fator que menos afeta a produtividade, desde que as plantas estejam distribuídas uniformemente 
na área (Endres, 1996). Por causa disto, a população de 400 mil plantas por hectare tem sido recomendada (Marcos Filho, 1986; Embrapa, 1993), embora outros fatores, como: região, época de semeadura, e cultivar utilizada, também influenciem a escolha da melhor população.

$\mathrm{O}$ espaçamento entre as linhas e a densidade de plantas nas linhas podem ser manipulados, com a finalidade de estabelecer o arranjo mais adequado à obtenção de maior produtividade e adaptação à colheita mecanizada. No arranjo onde o espaçamento entre linhas é igual ao espaçamento entre plantas dentro das linhas, têm sido observados aumentos na produtividade da soja (Moore, 1991; Ikeda, 1992; Egli, 1994); entretanto, o alto índice de mecanização da cultura, em todas as suas fases, impossibilita a adoção desse modelo de semeadura. Dessa forma, existe a necessidade de se fazer a semeadura em linhas, com espaçamentos bem definidos. Assim, os espaçamentos entre as linhas, utilizados na cultura da soja, variam entre 40 e $60 \mathrm{~cm}$ (Embrapa, 1997), e as maiores produtividades foram constatadas nos menores espaçamentos (Cardoso \& Rezende, 1987; Garcia, 1992).

Menores espaçamentos em uma mesma população proporcionam melhor distribuição espacial das plantas na área, com maior aproveitamento da radiação solar, pois permitem a redução da densidade de plantas nas linhas. Isto, de acordo com Ventimiglia et al. (1999), determina maior potencial de rendimento e produtividade real de grãos, o que justifica o aumento da produtividade obtida por alguns autores como Bullock et al. (1998).

Esta é uma tendência atual na cultura da soja, em que as densidades menores, em torno de 10 a 15 plantas $\mathrm{m}^{-1}$, vêm sendo utilizadas com sucesso, pois além de não reduzirem significativamente a produtividade, proporcionam redução nos custos de produção pela redução nos gastos com sementes. Segundo Peixoto (1998), as plantas de soja compensam a redução da densidade, por aumentarem a produção individual de legumes, o que contribui para maior tolerância a essa variação.

Além do arranjo mais adequado, a uniformidade de espaçamento entre as plantas distribuídas na linha também pode influir na produtividade dessa cultura. Plantas distribuídas de forma desuniforme im- plicam aproveitamento ineficiente dos recursos disponíveis, como luz, água e nutrientes. No caso da soja, o acúmulo de plantas em alguns pontos pode provocar o desenvolvimento de plantas mais altas, menos ramificadas, com menor produção individual, diâmetro de haste reduzido, e, portanto, mais propensas ao acamamento (Endres, 1996). Por outro lado, espaços vazios deixados na linha, além de facilitar o desenvolvimento de plantas daninhas, levam ao estabelecimento de plantas de soja com porte reduzido. $\mathrm{O}$ estande produzido dessa forma pode acarretar redução na produtividade, além das dificuldades por ocasião da colheita mecanizada.

O objetivo deste trabalho foi avaliar a influência do espaçamento entre linhas, da densidade de plantas nas linhas, e da uniformidade de semeadura, na produtividade e nas características agronômicas da soja.

\section{Material e Métodos}

Os ensaios foram realizados em Lavras, MG, latitude de $21^{\circ} 14^{\prime} \mathrm{S}$, longitude $45^{\circ} 0^{\prime} \mathrm{W}$ e altitude de $918 \mathrm{~m}$, num Latossolo Roxo distrófico, de textura argilosa fase cerrado no campo experimental do Departamento de Engenharia da Universidade Federal de Lavras (Ufla), nos anos agrícolas 1997/1998 e 1998/1999. As características químicas do solo no primeiro ano foram: $\mathrm{Ca}, 1,8 \mathrm{cmol}_{\mathrm{c}} \mathrm{dm}^{-3} ; \mathrm{Mg}$, $0,5 \mathrm{cmol}_{\mathrm{c}} \mathrm{dm}^{-3}$; Pe K, 10 e $101 \mathrm{mg} \mathrm{dm}^{-3}$, respectivamente, e no segundo ano: $\mathrm{Ca}, 1,9 \mathrm{cmol}_{\mathrm{c}} \mathrm{dm}^{-3} ; \mathrm{Mg}, 1,1 \mathrm{cmol}_{\mathrm{c}} \mathrm{dm}^{-3}$; $\mathrm{P}$ e K, 4 e $80 \mathrm{mg} \mathrm{dm}^{-3}$, respectivamente.

As semeaduras foram realizadas em 6/11/97 e 12/11/98, utilizando a cultivar CAC-1, recomendada para a região.

O delineamento experimental utilizado foi o de blocos ao acaso, com três repetições, em esquema de parcelas subsubdivididas. Os espaçamentos entre linhas, de $45 \mathrm{e}$ $60 \mathrm{~cm}$, foram dispostos nas parcelas, e as densidades de $10,13,16,19$ e 22 plantas $\mathrm{m}^{-1}$, nas subparcelas. Nas subsubparcelas, foram dispostos quatro níveis de uniformidade a saber: $25 \%, 35 \%, 60 \%$ e $100 \%$ das plantas espaçadas entre si. As subsubparcelas foram constituídas por quatro linhas de $5 \mathrm{~m}$ de comprimento, utilizando-se como área útil as duas linhas centrais menos os $0,5 \mathrm{~m}$ das extremidades.

Imediatamente antes da semeadura, as sementes foram submetidas à inoculação de Bradyrhizobium japonicum, na proporção de $1 \mathrm{~kg}$ de inoculante para $50 \mathrm{~kg}$ de sementes. No primeiro ano do experimento, foram aplicados 
$1.000 \mathrm{~kg} \mathrm{ha}^{-1}$ de calcário dolomítico, de acordo com as recomendações da Comissão de Fertilidade do Solo do Estado de Minas Gerais (1989). A adubação, conforme as recomendações da Comissão... (1989) para a cultura da soja, foi de $120 \mathrm{~kg} \mathrm{ha}^{-1}$ de $\mathrm{P}_{2} \mathrm{O}_{5}$ (superfosfato simples) e $20 \mathrm{~kg} \mathrm{ha}^{-1}$ de K (cloreto de potássio), distribuídos manualmente nos sulcos de semeadura.

A semeadura foi realizada manualmente, com o auxílio de réguas de madeira previamente perfuradas, de acordo com as densidades e níveis de uniformidade desejados. Para cada densidade, foram perfuradas quatro réguas, uma para cada nível de uniformidade. As uniformidades foram simuladas, considerando-se porcentagens de espaçamentos: "duplos" (D), menores que 0,5 vez o espaçamento médio esperado (Xref.); "aceitáveis" (A), de 0,5 a 1,5 vez o espaçamento médio esperado (Xref.); e "falhas" (F) maiores que 1,5 vez o espaçamento médio esperado (Xref.), conforme a Associação Brasileira de Normas Técnicas (1994), e o nível de uniformidade mencionado foi o relativo às porcentagens de espaçamentos aceitáveis. No nível de uniformidade de $100 \%$, todos os furos das réguas foram perfeitamente espaçados entre si (Xref.). Nos demais níveis de uniformidade, foram considerados 60\%(A), 20\%(D) e $20 \%(\mathrm{~F}) ; 35 \%(\mathrm{~A}), 30 \%(\mathrm{D})$ e $35 \%(\mathrm{~F}) ; 25 \%(\mathrm{~A}), 50 \%(\mathrm{D})$ e $25 \%(\mathrm{~F})$, ao realizar as furações das réguas. Ao lado de cada furo das réguas foram colocadas duas sementes, realizando-se, posteriormente, o desbaste, de forma a manter somente uma planta em cada posição desejada.

O controle de plantas daninhas foi realizado por meio da aplicação dos herbicidas Flex $\left(0,9 \mathrm{~L} \mathrm{ha}^{-1}\right)$ e Fusilade $\left(1,0 \mathrm{~L} \mathrm{ha}^{-1}\right)$, e o controle de lagarta-da-soja (Anticarsia gemmatalis), com a aplicação do inseticida Decis.

Foram avaliados, além da produtividade, os componentes da produção: número de legumes por planta, grãos por legume, e massa de 100 grãos e as características agronômicas, como: altura da planta e do primeiro legume, grau de acamamento, de acordo com Bernard et al. (1965), e porcentagem de sobrevivência.

A análise estatística dos dados foi realizada com auxílio do programa SAS - Statistics Analysis System - (SAS Institute,1999), e obedeceu ao modelo: $Y_{i j k l m}=\mu+a_{i}+$ $\mathrm{b}_{\mathrm{j}(\mathrm{i})}+\mathrm{E}_{\mathrm{k}}+\mathrm{aE}_{\mathrm{ik}}+\mathrm{e}_{\mathrm{ijk}}+\mathrm{D}_{\mathrm{l}}+\mathrm{aD}_{\mathrm{il}}+\mathrm{ED}_{\mathrm{kl}}+\mathrm{aED}_{\mathrm{ikl}}+\mathrm{e}_{\mathrm{ijkl}}+$ $\mathrm{U}_{\mathrm{m}}+\mathrm{aU}_{\mathrm{im}}+\mathrm{EU}_{\mathrm{km}}+\mathrm{DU}_{\mathrm{lm}}+\mathrm{aEU}_{\mathrm{ikm}}+\mathrm{aDU} \mathrm{ilm}_{\mathrm{m}}+\mathrm{EDU}_{\mathrm{klm}}+$ $\mathrm{aEDU}_{\mathrm{iklm}}+\mathrm{e}_{\mathrm{ijklm}}$ (Tourino, 2000), sendo: $\mathrm{i}=1,2, \mathrm{j}=1,2,3$, $\mathrm{k}=1,2,1=1,2,3,4,5, \mathrm{~m}=1,2,3,4 ; \mu$ : constante do modelo; $a_{i}$ : efeito do i-ésimo ano; $b_{j(i)}$ : efeito do j-ésimo bloco d do i-ésimo ano; $\mathrm{E}_{\mathrm{k}}$ : efeito do k-ésimo espaçamento; $\mathrm{aE}_{\mathrm{ik}}$ : efeito da interação do i-ésimo ano e k-ésimo espaçamento; $\mathrm{e}_{\mathrm{ijk}}$ : erro experimental na parcela; $\mathrm{D}_{1}$ : efeito da 1-ésima densidade; $\mathrm{aD}_{\mathrm{il}}$ : efeito da interação do i-ésimo ano e 1-ésima densidade; $E_{\mathrm{kl}}$ : efeito da interação do k-ésimo espaçamento e 1-ésima densidade; $\mathrm{aED}_{\mathrm{ikl}}$ : efeito da interação do i-ésimo ano, k-ésimo espaçamento e 1-ésima densidade; $\mathrm{e}_{\mathrm{ijk} k \mathrm{l}}$ : erro experimental a nível de subparcela; $\mathrm{U}_{\mathrm{m}}$ : efeito da m-ésima uniformidade; $\mathrm{aU}_{\mathrm{im}}$ : efeito da interação do i-ésimo ano e m-ésima uniformidade; $E_{\mathrm{km}}$ : efeito da interação do k-ésimo espaçamento e m-ésima uniformidade; $\mathrm{DU}_{\mathrm{lm}}$ : efeito da interação da l-ésima densidade e m-ésima uniformidade; $\mathrm{aEU}_{\mathrm{ikm}}$ : efeito da interação do i-ésimo ano, k-ésimo espaçamento e m-ésima uniformidade; $\mathrm{aDU}_{\mathrm{ilm}}$ : efeito da interação do i-ésimo ano, 1-ésima densidade e m-ésima uniformidade; EDU $\mathrm{klm}_{\mathrm{m}}$ : efeito da interação do k-ésimo espaçamento, 1-ésima densidade e m-ésima uniformidade;

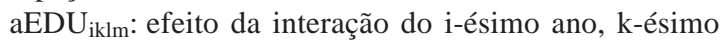
espaçamento, 1-ésima densidade e m-ésima uniformidade; $\mathrm{e}_{\mathrm{ijklm}}$ : erro experimental na subsubparcela.

De acordo com este modelo, os fatores principais foram testados com as suas interações com o fator ano. Quando os fatores densidade e uniformidade foram significativos, aplicou-se a análise de regressão para descrever as características avaliadas em função desses fatores.

\section{Resultados e Discussão}

Não houve efeito significativo dos fatores isolados, ou seja: espaçamento entre linhas, e densidade das plantas nas linhas, sobre a produtividade (Tabela 1). Houve, entretanto, comportamento diferenciado quanto à variação da densidade dentro de cada espaçamento (Tabela 2). No espaçamento de $45 \mathrm{~cm}$, o aumento da densidade de plantas provocou redução da produtividade, enquanto no espaçamento de $60 \mathrm{~cm}$, praticamente não houve resposta a esse fator, o que está de acordo com os resultados obtidos por Rezende et al. (1985). A maior disponibilidade de espaços nas entrelinhas no espaçamento de $60 \mathrm{~cm}$, pode ter compensado a redução dos espaços dentro das linhas, e houve maior emissão de ramos laterais. No menor espaçamento, esta compensação deve ter sido menor, e assim, o aumento da densidade causou maior competição entre as plantas, reduzindo a produtividade de cada planta. Segundo Marcos Filho (1986), a adaptação das plantas de soja aos espaços disponíveis justifica a falta de resposta em muitos experimentos que visam avaliar esses efeitos sobre a produtividade dessa leguminosa. 
Com espaçamento de $45 \mathrm{~cm}$ e densidade de 10 plantas $\mathrm{m}^{-1}$, ocorreu maior produtividade por área, possivelmente devido à melhor distribuição espacial das plantas, o que também contribuiu para o aumento da porcentagem de sua sobrevivência.

Em densidades menores, a produtividade por plan- ta aumenta, o que, segundo Garcia (1992), ocorre por causa da capacidade da soja em ajustar os componentes de produção. De fato, ocorreu um aumento na produção por planta com a redução da densidade (Tabelas 1 e 2). A maior produção por planta, neste caso, foi suficiente para superar a redução do núme-

Tabela 1. Médias de produtividade de grãos, altura da planta, grau de acamamento (GA), porcentagem de sobrevivência (PS), legumes por planta (LPP), grãos por legume (GPL) e massa de 100 grãos (M100). Ufla, Lavras, MG, 1997/1998 e 1998/1999(1).

\begin{tabular}{|c|c|c|c|c|c|c|c|c|}
\hline \multirow[t]{2}{*}{ Tratamento } & \multicolumn{2}{|c|}{ Grãos } & \multirow{2}{*}{$\begin{array}{l}\text { Altura de } \\
\text { planta }(\mathrm{cm})\end{array}$} & \multirow[t]{2}{*}{ GA } & \multirow{2}{*}{$\begin{array}{l}\text { PS } \\
(\%)\end{array}$} & \multirow[t]{2}{*}{ LPP } & \multirow[t]{2}{*}{ GPL } & \multirow{2}{*}{$\begin{array}{l}\text { M100 } \\
\text { (g) }\end{array}$} \\
\hline & $\mathrm{kg} \mathrm{ha}^{-1}$ & g planta $^{-1}$ & & & & & & \\
\hline \multicolumn{9}{|c|}{ Espaçamento $(\mathrm{cm})$} \\
\hline 45 & $2.244 \mathrm{a}$ & $7,28 \mathrm{~b}$ & $89 \mathrm{a}$ & $1,5 \mathrm{a}$ & $93 \mathrm{a}$ & $37 \mathrm{a}$ & $2,00 \mathrm{a}$ & $16,20 \mathrm{a}$ \\
\hline 60 & $2.239 \mathrm{a}$ & $9,53 \mathrm{a}$ & $89 \mathrm{a}$ & $1,5 \mathrm{a}$ & $94 \mathrm{a}$ & $46 \mathrm{a}$ & $2,05 \mathrm{a}$ & $16,00 \mathrm{a}$ \\
\hline \multicolumn{9}{|c|}{ Densidade (pl. $\mathrm{m}^{-1}$ ) } \\
\hline 10 & 2.309 & 12,16 & 84 & 1,1 & 98 & 51 & 2,09 & 15,74 \\
\hline 13 & 2.279 & 9,53 & 86 & 1,2 & 95 & 44 & 2,12 & 15,75 \\
\hline 16 & 2.250 & 7,86 & 91 & 1,6 & 93 & 39 & 2,05 & 16,29 \\
\hline 19 & 2.213 & 6,79 & 91 & 1,7 & 90 & 39 & 1,93 & 16,19 \\
\hline 22 & 2.156 & 5,68 & 92 & 1,9 & 91 & 34 & 1,93 & 16,53 \\
\hline \multicolumn{9}{|c|}{ Coeficientes das regressões significativas ${ }^{(2)}$} \\
\hline b & - & $-0,524$ & 0,74 & 0,070 & $-0,61$ & $-1,31$ & - & 0,067 \\
\hline c & - & 16,783 & 76,92 & 0,393 & 103,13 & 62,38 & - & 15,023 \\
\hline $\mathrm{R}^{2}$ & - & $0,96^{* *}$ & $0,89 * *$ & $0,97 *$ & $0,87 * *$ & $0,91 * *$ & - & $0,85^{* *}$ \\
\hline \multicolumn{9}{|c|}{ Uniformidade (\%) } \\
\hline 25 & 2.158 & 8,14 & 86 & 1,7 & 92 & 43 & 2,01 & 15,86 \\
\hline 35 & 2.232 & 8,25 & 89 & 1,5 & 94 & 42 & 2,01 & 16,12 \\
\hline 60 & 2.242 & 8,43 & 90 & 1,5 & 94 & 40 & 2,03 & 16,21 \\
\hline 100 & 2.334 & 8,79 & 90 & 1,3 & 94 & 41 & 2,06 & 16,19 \\
\hline \multicolumn{9}{|c|}{ Coeficientes das regressões significativas } \\
\hline $\mathrm{a}$ & - & - & - & - & - & - & - & $-0,0001$ \\
\hline b & 2,05 & 0,009 & - & $-0,0045$ & - & - & - & 0,0223 \\
\hline $\mathrm{c}$ & $2.128,7$ & 7,935 & - & 1,7625 & - & - & - & 15,447 \\
\hline $\mathrm{R}^{2}$ & $0,90 *$ & $1,00^{*}$ & - & $0,83 * *$ & - & - & - & $0,88^{*}$ \\
\hline Média & 2.241 & 8,40 & 89 & 1,5 & 93 & 41 & 2,03 & 16,10 \\
\hline
\end{tabular}

Tabela 2. Interação espaçamento entre linhas e densidade populacional de plantas nas linhas, sobre a produtividade e porcentagem de sobrevivência (PS). Ufla, Lavras, MG, 1997/1998 e 1998/1999.

\begin{tabular}{|c|c|c|c|c|c|c|c|c|}
\hline \multirow{4}{*}{$\begin{array}{l}\text { Densidade } \\
\text { de plantas } \\
\left(\mathrm{pl} . \mathrm{m}^{-1}\right)\end{array}$} & \multicolumn{8}{|c|}{ Espaçamento entre linhas $(\mathrm{cm})$} \\
\hline & \multicolumn{4}{|c|}{45} & \multicolumn{4}{|c|}{60} \\
\hline & \multirow{2}{*}{$\begin{array}{c}\text { População } \\
\text { (x 1.000) }\end{array}$} & \multirow{2}{*}{$\begin{array}{l}\text { PS } \\
(\%)\end{array}$} & \multicolumn{2}{|c|}{ Produtividade } & \multirow{2}{*}{$\begin{array}{r}\text { População } \\
\text { (x 1.000) }\end{array}$} & \multirow{2}{*}{$\begin{array}{l}\text { PS } \\
(\%)\end{array}$} & \multicolumn{2}{|c|}{ Produtividade } \\
\hline & & & $\left(\mathrm{kg} \mathrm{ha}^{-1}\right)$ & $\left(\right.$ g planta $\left.^{-1}\right)$ & & & $\left(\mathrm{kg} \mathrm{ha}^{-1}\right)$ & $\left(\right.$ g planta $\left.^{-1}\right)$ \\
\hline 10 & 222 & 98 & 2.374 & 10,75 & 167 & 97 & 2.243 & 13,57 \\
\hline 13 & 289 & 95 & 2.295 & 8,25 & 217 & 95 & 2.264 & 10,82 \\
\hline 16 & 356 & 93 & 2.242 & 6,76 & 267 & 94 & 2.258 & 8,97 \\
\hline 19 & 422 & 90 & 2.243 & 5,95 & 317 & 90 & 2.183 & 7,62 \\
\hline 22 & 489 & 90 & 2.065 & 4,67 & 367 & 92 & 2.248 & 6,69 \\
\hline Média & & 93 & 2.244 & 7,28 & & 94 & 2.239 & 9,53 \\
\hline
\end{tabular}


ro de plantas nas linhas, mantendo os níveis de produtividade, no espaçamento $0,60 \mathrm{~m}$, e até aumentando-a na densidade menor, com espaçamento de $0,45 \mathrm{~m}$. Para a cultivar utilizada, portanto, é possível a redução da densidade para 10 plantas $\mathrm{m}^{-1}$, mesmo porque durante os dois anos de observação não foi constatado aumento na infestação de plantas daninhas, graças a este fator. Estes resultados possibilitam uma economia de mais de $100 \%$ de sementes, em comparação com a densidade de 22 plantas $\mathrm{m}^{-1}$.

Nas menores densidades, as plantas foram mais baixas $(\mathrm{P}<0,01)$, acamaram menos $(\mathrm{P}<0,05)$, e, portanto, foram menos sujeitas aos efeitos negativos desse fator, resultando em maior porcentagem de sobrevivência $(\mathrm{P}<0,01)$, o que também pode ter contribuído para o aumento da produtividade.

Com relação à uniformidade de semeadura, a análise de variância detectou efeito significativo sobre a produtividade a $8 \%$ de probabilidade. A análise de regressão revelou ainda efeito linear significativo quanto à produtividade e com relação à produtividade por planta (Tabela 1). Resultados semelhantes foram obtidos por Torres \& Garcia (1991), segundo os quais, a falta de uniformidade provocada pelas falhas na semeadura afeta negativamente a produtividade da soja. Houve aumento médio, em dois anos, de $176 \mathrm{~kg} \mathrm{ha}^{-1}(8,2 \%)$, e de 0,65 g planta $^{-1}(8,0 \%)$, com o aumento da uniformidade de 25 para $100 \%$ (Tabela 1). De acordo com a equação da reta ajustada para a produtividade por área era esperado um aumento médio de $154 \mathrm{~kg} \mathrm{ha}^{-1}$, devido a esta variação na uniformidade. Resultados, estes, que concordam com Moore (1991) - o qual obteve aumento de produtividade, $157 \mathrm{~kg} \mathrm{ha}^{-1}$ no primeiro ano, e $257 \mathrm{~kg} \mathrm{ha}^{-1}$ no segundo ano quando as plantas de soja tiveram espaçamento uniforme -, e com Egli (1994), que observou aumentos de 9 a $19 \%$ na produtividade da soja, no caso de uniformidade de espaçamentos. Esses resultados sugerem que a operação de semeadura da soja com maior precisão na deposição das sementes no solo é um aspecto relevante, pois pode contribuir para o aumento da sua produtividade.

Reduções na uniformidade acima de $50 \%$ podem proporcionar perdas em torno de $103 \mathrm{~kg} \mathrm{ha}^{-1}$, o que é uma perda significativa, considerando-se que a sojicultura está inserida em um mercado extremamente competitivo, com reduzidas margens de lucro.
Dessa forma, qualquer fator que aumente o nível de produtividade deve ser considerado.

Na menor densidade (10 plantas $\left.\mathrm{m}^{-1}\right)$, observouse maior efeito da uniformidade entre plantas dentro das linhas (Figura 1). O aumento da uniformidade, neste caso, proporcionou aumento de até $400 \mathrm{~kg} \mathrm{ha}^{-1}$, e as maiores respostas à densidade das plantas nas linhas ocorreram quando as plantas foram plantadas a espaços uniformes acima de $60 \%$. As maiores produtividades, contidas no intervalo de 2.400 a $2.500 \mathrm{~kg} \mathrm{ha}^{-1}$, foram obtidas dentro da superfície delimitada pela uniformidade de $60 \%$ a $100 \%$ e pela densidade de 10 a 13 plantas $\mathrm{m}^{-1}$. Assim, a utilização de menores densidades com plantas uniformemente distribuídas nas linhas permite maior expressão do potencial produtivo das plantas, pelo melhor aproveitamento dos fatores do meio e dos insumos aplicados. Entretanto, a uniformidade de semeadura de soja acima de $60 \%$ dificilmente é atingida pelos sistemas dosadores das semeadoras tradicionais, com princípios mecânicos, o que indica a necessidade de aprimoramento desses sistemas, ou a utilização de sistemas mais precisos, como, por exemplo, os pneumáti-

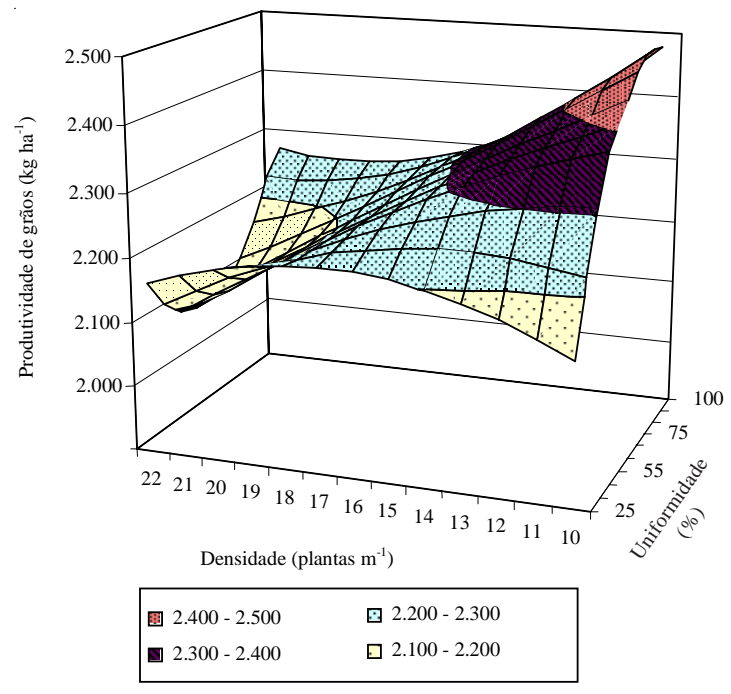

Figura 1. Superfície de resposta referente a produtividade (PROD) de soja, média de dois anos, em razão da densidade (D) de plantas nas linhas, e uniformidade (U) de espaçamento entre plantas dentro das linhas. Ufla, Lavras, MG, 1997/1998 e 1998/1999. (PROD = 785,06 + 122,74D + $37,33 \mathrm{U}-2,32 \mathrm{D}^{2}-0,13 \mathrm{U}^{2}-2,17 \mathrm{DU}+0,00047 \mathrm{D}^{2} \mathrm{U}^{2}$; $\left.R^{2}=0,63\right)$. 
cos, como fator importante para o aumento da produtividade da cultura.

Quando a uniformidade foi reduzida de $100 \%$ para $25 \%$, o aumento da porcentagem de espaçamentos duplos de $0 \%$ para $50 \%$ provocou o acúmulo de plantas em determinados pontos das linhas. Nestes pontos, as plantas foram mais altas. Na média, a altura das plantas desses tratamentos foi menor, pois foi compensada pelas alturas das plantas mais baixas localizadas nos pontos de menor concentração. As plantas mais altas, entretanto, tenderam a acamar mais, elevando o grau de acamamento desses tratamentos. Com o aumento da uniformidade isso não ocorreu, pois a altura das plantas foi mais uniforme, e resultou em redução significativa no grau de acamamento (Tabela 1). Assim, a redução do acamamento obtida com o aumento da uniformidade é fator importante a considerar, uma vez que plantas acamadas dificultam a colheita mecanizada e pode proporcionar perdas consideráveis.

O número de legumes por planta variou significativamente $(\mathrm{P}<0,06)$, por causa do espaçamento, e ocorreu aumento nessa característica no espaçamento de $60 \mathrm{~cm}$, provavelmente devido à maior emissão de ramos laterais pelas plantas, o que está de acordo com Marcos Filho (1986). Não foram, entretanto, detectadas diferenças significativas no número de grãos por legume e massa de 100 grãos em decorrência dessa característica. A ausência de resposta da massa de grãos à variação do espaçamento foi relatada também por Cardoso \& Rezende (1987).

A variação da densidade afetou significativamente o número de legumes por planta e a massa de 100 grãos, e não houve efeito significativo sobre o número de grãos por legume (Tabela 1). O número de legumes por planta variou inversamente à variação da densidade das plantas na linhas, ou seja, a redução da densidade provocou aumento no número de legumes por planta. Resultados semelhantes foram relatados por Garcia (1992), Carpenter \& Board (1997) e Peixoto (1998). A adaptação aos espaços disponíveis, com emissão de maior número de ramificações à medida que aumentam esses espaços pode causar essas variações. Neste caso, o aumento do número de legumes por planta, na cultivar utilizada, foi suficiente para compensar a redução do número de plantas por metro linear (densidade), e ainda proporcionou aumento da produtividade de grãos. A massa de 100 grãos, por sua vez, aumentou com o aumento da densidade, o que está de acordo com os resultados de Peixoto (1998). A análise de regressão realizada, no que se refere ao número de legumes por planta e massa de 100 grãos em razão da densidade, foi significativa em relação a ambas as características. Para cada planta acrescentada na densidade, há uma redução média de 1,31 legume por planta e um aumento de 0,067 g na massa de 100 grãos, indicando, assim, que com a redução do número de legumes competindo por fotoassimilados houve maior concentração destes nos grãos.

Não houve efeito da variação na uniformidade de semeadura sobre o número de legumes por planta e número de grãos por legume (Tabela 1). Esses resultados estão de acordo com os obtidos por Ikeda (1992), no que se refere a número de grãos por legume. Observa-se, entretanto, uma leve tendência do número de grãos por legume de aumentar com a uniformidade de espaçamento entre plantas nas linhas.

A massa de 100 grãos variou significativamente, com efeito quadrático, com a variação da uniformidade de semeadura (Tabela 1). Houve um aumento nessa característica com o aumento da uniformidade, o qual foi mais acentuado entre as uniformidades de 25 e $35 \%$. Provavelmente, este aumento ocorreu, em virtude do aumento da eficiência de utilização da energia solar, que com o aumento da uniformidade foi melhor distribuída sobre as folhas, resultando em maior concentração de assimilados nos grãos. Estes resultados estão de acordo com os obtidos por Moore (1991), que, utilizando três densidades, em dois anos, observou aumento no peso e no tamanho das sementes com o aumento da uniformidade.

\section{Conclusões}

1. A produtividade da soja aumenta com a redução do espaçamento entre linhas aliado à redução da densidade de plantas nas linhas; o espaçamento de $45 \mathrm{~cm}$ com a densidade de 10 plantas $\mathrm{m}^{-1}$ proporciona melhor distribuição das plantas na área, permitindo, graças às alterações na sua arquitetura, um maior fechamento das entre linhas, e portanto, melhor controle de plantas daninhas. 
2. Nas menores densidades, as plantas são mais baixas, acamam menos, e apresentam maior porcentagem de sobrevivência.

3. O aumento da uniformidade de espaçamento entre plantas dentro das linhas contribui para a redução do acamamento, e para o aumento da produtividade da soja.

\section{Referências}

ASSOCIAÇÃO BRASILEIRA DE NORMAS TÉCNICAS (Rio de Janeiro, RJ). Projeto de norma 04:015.06-004 - semeadoras de precisão: ensaio de laboratório - método de ensaio. São Paulo, 1994. 26 p.

BERNARD, R. L.; CHAMBERLAIN, D. W.; LAWRENCE, R. D. (Ed.). Results of the cooperative uniform soybean tests. Washington: Usda, 1965. $134 \mathrm{p}$.

BULLOCK, D.; KHAN, S.; RAYBURN, A. Soybean yield response to narrow rows is largely due to enhanced early growth. Crop Science, Madison, v. 38, n. 4, p. 1011-1016, 1998.

CARDOSO, D. A. D. B.; REZENDE, P. M. de. Arranjo de plantas. I. Efeito do espaçamento e da densidade no rendimento de grãos e outras características da soja. Ciência e Prática, Lavras, v. 11, n. 1, p. 23-33, 1987.

CARPENTER, A. C.; BOARD, J. E. Branch yield components controlling soybean yield stability across plant populations. Crop Science, Madison, v. 37, n. 5, p. 1520 1526, 1997.

COMISSÃO DE FERTILIDADE DO SOLO DO ESTADO DE MINAS GERAIS (Lavras, MG). Recomendações para o uso de corretivos e fertilizantes em Minas Gerais. Lavras, 1989. 159 p.

EGLI, D. B. Mechanisms responsible for soybean yield response to equidistant planting patterns. Agronomy Journal, Madison, v. 86, n. 6, p. 1046-1049, 1994.

EMBRAPA. Centro Nacional de Pesquisa de Soja (Londrina, PR). Recomendações técnicas para a cultura da soja na região central do Brasil: 1993/1994. Londrina, 1993. 120 p. (Documentos, 64).

EMBRAPA. Centro Nacional de Pesquisa de Soja (Londrina, PR). Recomendações técnicas para a cultura da soja na região central do Brasil: 1997/1998. Londrina, 1997. 171 p. (Documentos, 106).
EMBRAPA. Centro Nacional de Pesquisa de Soja (Londrina, PR). Recomendações técnicas para a cultura da soja no Paraná: safra 2000/2001. Londrina, 2000. 255 p.

ENDRES, V. C. Espaçamento, densidade e época de semeadura. In: EMBRAPA. Centro de Pesquisa Agropecuária do Oeste (Dourados, MS). Soja: recomendações técnicas para Mato Grosso do Sul e Mato Grosso. Dourados, 1996. p. 82-85. (Circular Técnica, 3).

GARCIA, A. Manejo da cultura da soja para alta produtividade. In: SIMPÓSIO SOBRE CULTURA E PRODUTIVIDADE DA SOJA, 1., 1991, Piracicaba. Anais... Piracicaba: Fealq, 1992. p. 213-235.

IKEDA, T. Soybean planting patterns in relation to yield and yield components. Agronomy Journal, Madison, v. 84, n. 6, p. 923-926, 1992.

MARCOS FILHO, J. Produção de sementes de soja. Campinas: Fundação Cargill, 1986. 86 p.

MOORE, S. H. Uniformity of planting effect on soybean population parameters. Crop Science, Madison, v. 31, n. 4, p. 1049-1051, 1991.

PEIXOTO, C. P. Análise de crescimento e rendimento de três cultivares de soja em três épocas de semeadura e três densidades de plantio. 1998. $151 \mathrm{f}$. Tese (Doutorado) - Escola Superior de Agricultura Luiz de Queiroz, Piracicaba.

REZENDE, P. M. de; VIEIRA, M. das G. G. C.; FRAGA, A. C.; FAVORETO, C. R. S. Efeitos da densidade de plantas, sobre a produção, qualidade das sementes e outras características da soja [Glycine $\max (\mathrm{L}$.) Merrill]. Ciência e Prática, Lavras, v. 9, n. 1, p. 30-38, 1985.

SAS INSTITUTE (Cary, Estados Unidos). SAS procedures guide for computers. 6 . ed. Cary, 1999. v. 3.

TORRES, E.; GARCIA, A. Uniformidade de distribuição de plantas em lavouras de soja. Londrina: EmbrapaCNPSo, 1991. 9 p. (Comunicado Técnico, 48).

TOURINO, M. C. C. Arranjo populacional e uniformidade de semeadura na produtividade e outras características agronômicas da soja [Glycine max (L.) Merrill]. 2000. 139 f. Tese (Doutorado) - Universidade Federal de Lavras, Lavras.

VENTIMIGLIA, L. A.; COSTA, J. A.; THOMAS, A. L.; PIRES, J. L. F. Potencial de rendimento da soja em razão da disponibilidade de fósforo no solo e dos espaçamentos. Pesquisa Agropecuária Brasileira, Brasília, v. 34, n. 2, p. 195-199, fev. 1999. 